\title{
Tecnologías de Información y Comunicación como Estrategia de Apropiación de la Lectura en Niños de Primaria
}

\section{Information and Communication Technologies As a Strategy for the Appropriation of Reading in Primary School Children}

\author{
Heidis Padilla-Domínguez \\ heidisbeatriz@gmail.com \\ Institución Educativa Técnica Agropecuaria Santa Bárbara \\ Colombia \\ https://orcid.org/0000-0003-0890-3565
}

Recibido: 25 de noviembre de 2019

Aprobado: 20 de diciembre de 2019

\begin{abstract}
RESUMEN
El presente artículo tuvo como propósito analizar las TIC como estrategia de Apropiación de la Lectura en Niños de Primaria. El mismo fue sustentado con las teorías de Piñeros y Patiño (2013), Cristóbal (2011), Molano, Quiroga, Romero y Pinilla (2014), Ramos (2013), Landazábal (2011) López (2012). Yanes (2011), Hepp (2011), MEN (2013), Morrissey (2011), Lugo y Kelly (2011) Martín (2011), Landazábal (2011), entre otros. Se desarrolló bajo la metodología del análisis documental enmarcada en el paradigma cualitativo. En ese sentido, se realizó un análisis documental revisando documentos científicos para identificar y analizar aspectos asociados a las TIC como estrategia de Apropiación para el aprendizaje de la lectura. Se concluyó que el empleo de las TIC como estrategia de Apropiación de la Lectura en Niños de Primaria requiere tanto de la gestión de recursos tecnológicos como de la capacitación docente para el uso efectivo de los mismos.
\end{abstract}

Descriptores: TIC; Aprendizaje; Estrategia; Lectura; Educación Inicial.

\begin{abstract}
The purpose of this article was to analyze the ICT as a strategy for the Appropriation of Reading in Primary Children. It was supported by the theories of Piñeros and Patiño (2013), Cristóbal (2011), Molano, Quiroga, Romero and Pinilla (2014), Ramos (2013), Landazábal (2011) López (2012). Yanes (2011), Hepp (2011), MEN (2013), Morrissey (2011), Lugo and Kelly (2011) Martín (2011), Landazábal (2011), among others. It was developed under the methodology of documentary analysis framed in the qualitative paradigm. In that sense, a documentary analysis was carried out
\end{abstract}




\author{
CIENCIAMATRIA \\ Revista Interdisciplinaria de Humanidades, Educación, Ciencia y Tecnología \\ Año VI. Vol. VI. N${ }^{\circ}$. Edición Especial. 2020 \\ Hecho el depósito de ley: pp201602FA4721 \\ ISSN-L: 2542-3029; ISSN: 2610-802X \\ Universidad Nacional Experimental Francisco de Miranda (UNEFM). Santa Ana de Coro. Venezuela
}

Heidis Padilla-Domínguez

reviewing scientific documents to identify and analyze aspects associated with ICT as a strategy of Appropriation for the learning of reading. It was concluded that the use of ICT as a strategy for the Appropriation of Reading in Primary School Children requires both the management of technological resources and teacher training for their effective use.

Descriptors: TIC; Learning; Strategy; Reading; Initial education.

\title{
INTRODUCCIÓN
}

A nivel mundial el siglo XXI trajo consigo una rebelión tecnológica que ha logrado impactar drásticamente el estilo de vida de los seres humanos al incorporar las tecnologías de Información y Comunicación (TIC). De allí entonces, que dicho fenómeno está estimulando la fusión de diversas culturas a nivel mundial en una sola cultura global; es decir, ha generado el denominado fenómeno de la globalización.

Lo anterior expuesto, va de la mano o ha servido de estímulo al surgimiento de una nueva sociedad; llamada sociedad del conocimiento, lo cual según la UNESCO (2013), advierte a los sistemas educativos del mundo una imperante necesidad de actualizarse y adaptar sus prácticas y contenidos a la naciente sociedad de la información.

A raíz de todo este proceso de adaptación antes mencionado pudiera implicar un doble desafío; por una parte, se necesita atender aspectos relacionados con la capacitación docente para incorporar las TIC al aula y en el currículum escolar; y por la otra, está el de centrar la atención en el diseño, implantación y evaluación de políticas públicas que se supone garantizarían su implementación de manera sistémica. De la misma manera se hace pertinente materializar dichas políticas de manera que se evidencie una reforma integral al hecho educativo, considerando la cobertura y calidad de la infraestructura tecnológica (hardware, software y acceso a servicios de información y comunicación).

Sobre el particular puede señalarse que los sistemas educativos del mundo se enfrentan al reto de transformarse para evolucionar. No obstante, a juicio de Yanes (2011), el proceso de evolución pudiera no ser tan sencillo, puesto que se trata de 
romper con todos los paradigmas educativos que anteriormente servían a una sociedad industrial para abrir paso a nuevas prácticas pedagógicas que faciliten la adaptación de los seres humanos a la nueva sociedad del conocimiento.

Lo expresado por el autor en cuestión, si bien no pareciera ser nada fácil, tampoco debería ser considerado como propósito inalcanzable, teniendo en cuenta que de lo que se trata es de emplear los métodos y estrategias más adecuados para preparar a los estudiantes, de manera que puedan ser independientes a la hora de renovar sus cocimientos para asumir el desafío de autoformarse integralmente.

En ese marco de acción, pudiera hacerse realidad el desarrollo de habilidades y competencias a partir de la innovación en los procesos de mediación que facilitan el acceso a las TIC, para que los estudiantes logren apropiarse de las habilidades básicas de lectura, escritura; las relacionadas con las matemáticas; y al mismo tiempo, aprendan a resolver problemas; desarrollen el pensamiento crítico, la creatividad y la productividad en un clima de trabajo colaborativo y ciudadanía responsable.

En el mismo orden de ideas, la Sociedad Internacional para la Tecnología en Educación, conocida por su sigla en inglés ISTE (2011) reconoce que muchos docentes no tienen las competencias necesarias para diseñar e implementar ambientes de aprendizaje ricos en tecnología. MEN (2013), esta situación pudiera tener su raíz en el miedo a asumir la demanda de nuevos paradigmas educativos que muchos docentes experimentan al no sentirse preparados para enfrentarse a situaciones desconocidas que en materia de mediación tecnológica puedan surgir.

Así pues, por ejemplo, en el área de lenguaje, muchas de las prácticas educativas que se llevan a cabo para que los niños se apropien de la lectura y escritura, son memorísticas y poco atractivas para captar la atención del curso y lograr un impacto que redunde en la adquisición adecuada de éstas habilidades.

De allí que surge la necesidad de enfocarse hacia practicas educativas innovadoras, que apunten al uso de las TIC, las cuales deben facilitar en los estudiantes, el inicio del aprendizaje de la lectura y escritura de una manera significativa, de tal manera 
que los procesos que se inicien en estas habilidades sean sólidos, para así convertirse en un pilar fundamental para la adquisición de nuevos conocimientos.

Surge entonces la gran preocupación a nivel mundial de cómo se desarrolla la educación básica primaria, por la importancia que ésta tiene en los inicios de la formación de los estudiantes, al respecto, Delors J. (1.997 p.20) afirma que "la educación básica es un problema que se plantea, lógicamente, en todos los países, incluidos los industrializados". Desde este nivel de la educación, los contenidos que se desarrollen deben buscar fomentar el deseo de aprender, el ansia y la alegría de conocer y por lo tanto, el afán y las posibilidades de acceder más tarde a la educación durante toda la vida.

Desde esa perspectiva surge entonces la necesidad de recalcar el papel fundamental que juega la práctica educativa del docente en el aprendizaje de los estudiantes, entendiendo que la misma debe estimularse con estrategias tecnológicas atractivas y significativas donde se genere en dichos estudiantes ese deseo de aprender y así poder apropiarse de las herramientas necesarias para construir su conocimiento.

\section{DESARROLLO TEÓRICO}

Para la construcción teórica del presente artículo fue necesario llevar a cabo un proceso de reflexión, análisis crítico y ajustes progresivos, donde por medio de la investigación e indagación del docente se puedan construir nuevas realidades que estimulen a los estudiantes a utilizar las TIC como estrategia de apropiación en la lectura en la que se internalice en ellos la frase que señala que para exigirse y avanzar en la sociedad es necesario no tanto tener más sino ser más, pues ésta es la verdadera condición del progreso humano.

\section{Las TIC como estrategia de Apropiación de la lectura}

Las constantes innovaciones y los acelerados cambios en las tecnologías de la información y las comunicaciones (TIC), a través del internet, facilitan la disponibilidad de grandes volúmenes de datos que pueden ser compartidos en todo el mundo de manera rápida y eficaz. Estos beneficios han creado las condiciones 


\section{Heidis Padilla-Domínguez}

necesarias para el surgimiento de una nueva cultura global en la cual se encuentra inmersa la llamada sociedad del conocimiento, entendida como el conjunto de personas que han aprendido a procesar rápidamente la información a fin de adaptarse al dinamismo de su entorno.

Asimismo, Hepp (2011, p. 75) en esa misma línea de pensamiento destaca que las TIC representan una "herramienta eficaz que permite preparar a los jóvenes para adaptarse a la dinámica de la sociedad informacional, permitiéndoles desarrollar habilidades para la vida en relación con el manejo de información y la comunicación con otras personas". En este sentido, la incorporación de las TIC en los centros educativos hace posible que sus estudiantes puedan conectarse con el mundo aprovechado las ventajas y recursos educativos del internet, además estos pueden interactuar con personas de diversas culturas mediante la música, juegos, espacios sociales, producción de contenidos, entre otros.

El surgimiento de dicha sociedad impacta de forma directa los sistemas formales de educación, obligándolos a replantearse el hecho educativo de cara a una nueva demanda social en la materia. Ello implica la formulación e implantación de nuevos métodos para la mediación de los aprendizajes que consideren las TIC, definidas por la UNESCO, como el "conjunto de disciplinas científicas, tecnológicas, de ingeniería y de técnicas de gestión utilizadas en el manejo y procesamiento de la información; sus aplicaciones; los computadores y su interacción con hombres y máquinas; y los contenidos asociados de carácter social, económico y cultural". (Yanes 2011, p. 33). Asimismo, la incorporación de estas tecnologías representa una acción estratégica que a juicio de Tobón (2011, p. 272) estimula el desarrollo de las inteligencias múltiples en los sujetos mediados y a su vez "posibilitan diseñar y articular las actividades docentes teniendo en cuenta los ritmos de aprendizajes de los estudiantes y sus diferentes potencialidades". Desde esta perspectiva, se conciben las TIC como una estrategia de mediación para el desarrollo del potencial cognitivo de las personas, y específicamente para el aprendizaje de la lectura.

En efecto, para Salas (2007) citado por Molano, Quiroga, Romero y Pinilla (2014, p. 8), la lectura es un proceso donde "se adquieren habilidades de carácter cognitivo, 
afectivo y conductual, que debe ser manejado estratégicamente por etapas", ello implica el desarrollo de diversas estrategias con propósitos específicos dentro del mismo proceso lector.

Por tanto, las TIC como estrategia de mediación para el aprendizaje de la lectura en educación primaria, se concibe como un proceso mediante el cual se gestionan los ambientes tecnológicos que favorecen la mediación para el aprendizaje lector, a fin de aprovechar estos espacios en cada memento de dicha mediación (explorador, integrador e innovador) considerando los métodos de lectura seleccionados (sintético, analítico y mixto); y las etapas del proceso lector donde se encuentre el sujeto mediado (adquisición, uso y desarrollo de la lectura).

Por otra parte, según su capacidad de estímulo cognitivo al sujeto mediado, los ambientes tecnológicos que favorecen la mediación para el aprendizaje lector pueden ser clasificados en, audiovisuales, virtuales e interactivos. Estos ambientes, según el MEN (2013, p. 16), requieren ser gestionados mediante la dotación de equipos y conectividad a las instituciones educativas colombianas.

No obstante, su incorporación no garantiza el uso pedagógico correcto de los mismos, por tanto, las TIC como estrategia de mediación en las instituciones educativas requieren del abordaje de otros aspectos relacionados con la creación de una cultura tecnológica que implica el desarrollo de procesos de capacitación docente para su incorporación en los procesos de planificación, formación y evaluación de los aprendizajes.

\section{Revolución digital y lecto-escritura}

Hay perfecto acuerdo entre los especialistas de hablar de una revolución digital, de una "cultura digital". Como bien señala Pérez (2006, p.23) dicha expresión no alude a que toda la cultura se haya digitalizado, "sino que el digitalismo marca la pauta de la cultura en su conjunto como un sector emergente de ella. El digitalismo es el resultado dinámico del desarrollo y expansión de las nuevas tecnologías de la información y la comunicación, las cuales inciden fuertemente en la sociedad y en la cultura desde las que nacen modificándolas a su vez profundamente".

En el mismo orden de ideas, señala Gros, (2010, p.20) que esta revolución 


\section{Heidis Padilla-Domínguez}

tecnológica, esta sustitución del átomo por el bit, produce una profunda transformación de la esencia misma del ser humano, el cual está evolucionando, a un ritmo exponencial, de homo sapiens a homo digitalis, con todas las consecuencias, que en todos los campos de la vida humana, ello implica y en y con lo cual ya estamos inmerso, no siendo el campo de la educación una excepción.

En palabras de Sáez (2004), esta revolución tecnológica está produciendo una noomorfis ("formación de la inteligencia" p.45), un cambio de las estructuras mentales y la forma misma de la inteligencia que se está operando fundamentalmente entre los llamados nativos digitales como consecuencia de su temprana e intensiva inmersión en una infoestructura cada vez más densa y extensa, denominada, por el citado autor, Red Universal Digital: "conjunto heterogéneo de todas las redes, un organismo electrónico gigantesco por su extensión y operativamente invisible, que está creciendo a nuestro alrededor y soportando cada día más funciones sociales, sin que apenas seamos conscientes de su realidad y sobre todo de su magnitud".

\section{Ambientes Tecnológicos que Favorecen la Apropiación para el Aprendizaje Lector}

Los ambientes tecnológicos que favorecen la mediación para el aprendizaje lector (audiovisual, virtuales e interactivos) son espacios que ofrecen una gran variedad de estímulos visuales, auditivos, cognitivos e interactivos, que pueden ser aprovechados para el aprendizaje de la lectura. Estos son considerados en cada momento de la mediación tecnológica (explorador, integrador e innovador) haciendo más efectivos los métodos empleados para el aprendizaje lector (Sintético, analítico y mixto) y facilitando el aprendizaje de la lectura en cualquiera de sus etapas (adquisición, uso y desarrollo).

Asimismo, las bondades que ofrecen estos ambientes son reconocidas a nivel mundial, de allí que la UNESCO - OREALC, (2007) citado por MEN (2013, p. 49) considera que "el desarrollo profesional docente debe articularse con esfuerzos complementarios en dotación de infraestructura tecnológica, diseño de materiales educativos, construcción de propuestas didácticas innovadoras, gestión escolar, 


\section{Heidis Padilla-Domínguez}

adaptación curricular y construcción de modelos de evaluación”. A la luz de estas afirmaciones, se entiende que dichas bondades están determinadas por diversos factores que posibilitan su aprovechamiento, los cuales deben ser analizados y gestionados según la naturaleza del entorno educativo.

Por otra parte, el MEN (2013) considera que un ambiente innovador más allá de ser concebido como un espacio donde se usan las TIC, debe conceptualizarse mediante criterios que ofrezcan una perspectiva más amplia e integral relacionada con la posibilidad que ofrecen estos espacios para que los estudiantes desarrollen pensamiento crítico, autónomo y creativo; a través del trabajo en equipo y con la utilización de las nuevas tecnologías.

A las anteriores ideas se suman las consideraciones de Hepp (2011) quien destaca que aunque ciertamente no se puede generalizar en cuanto a los aportes de las TIC en los aprendizajes, es un hecho notorio que las mismas enriquecen los ambientes de aprendizaje haciéndolos más atractivos y pertinentes para los estudiantes. En estos planteamientos se vislumbran dos reflexiones importantes; la primera está relacionada con el potencial de estos recursos ofrecen para el desarrollo de la lectura independientemente de que sean o no aprovechados; la segunda tiene que ver con la importancia del proceso de mediación tecnológica como hecho determinante en el aprovechamiento de los mismos.

En este orden de ideas, Lugo y Kelly (2011) consideran que el aprovechamiento de los ambientes tecnológicos que favorecen la mediación para el aprendizaje lector debe empezar por distinguir entre recursos tecnológicos e infraestructura tecnológica. Destaca además el mencionado autor que se denominan recursos tecnológicos a la diversidad de material de aprendizaje en formato digital y las herramientas de producción con las que interactúa docentes y estudiantes en las instituciones educativas. Asimismo, a diferencia de los recursos, la infraestructura tecnológica está conformada por la dotación de espacios con computadoras, proyectores, escáner e impresoras, redes y servidores con conexión a internet y demás medios tecnológicos que pueden ser utilizados en los procesos de mediación. 


\section{METODOLOGÍA}

En este aspecto, se definen los procedimientos que se realizan para abordar la investigación, al respecto, Mercado (2010 p.2) explica que: "Es el conjunto de procedimientos (métodos y técnicas) que se aplican para responder al problema de investigación".

En el mismo sentido, el enfoque usado es el cualitativo debido a que en la presente investigación se estudia acciones humanas, tales como las prácticas educativas que involucran tanto a docentes como estudiantes; con relación a esto, Rodríguez (2013 p. 20) afirma que el objeto de estudio del enfoque cualitativo son "hechos subjetivos relativos a la comprensión del significado de las acciones humanas", por tanto usando este método es posible ahondar en el proceso de enseñanza, permitiendo conocer de mejor manera de qué forma se está realizando éste en la educación del grado primero y poder así reconocer que estrategias se pueden implementar para que se desarrolle de manera exitosa; logrando el fin último de que los estudiantes se apropien de la lectura de una manera significativa y agradable. Como también lo señala López (2019) cuando expresa que los datos cualitativos son aquellos en los cuales se utilizan diversas categorías temáticas al momento de analizar su contenido.

En el mismo sentido se utilizó el análisis documental, el cual según Castillo (2014) explica que es una operación intelectual que da lugar a un subproducto o documento secundario que actúa como intermediario o instrumento de búsqueda obligado entre el documento original y el usuario que solicita información. El calificativo de intelectual se debe a que el documentalista debe realizar un proceso de interpretación y análisis de la información de los documentos y luego sintetizarlo.

Es así, que el proceso antes mencionado se desplegó considerando un paradigma, el cual es definido por estos autores, como la perspectiva contextual del investigador con el cual se aborda el fenómeno, para responder así las interrogantes planteadas durante el diseño preliminar del estudio; otro de los elementos que se consideró, fue la generación de marcos conceptuales, que a manera similar como en la codificación abierta, representa la manera como se relacionan y se activan para categoría. 
En ese sentido, el uso adecuado de lo que hasta ahora se desarrolló, es importante, puesto que para los autores representan la guía donde confluyen los aspectos conceptuales y contextuales para la comparación sistemática entre las categorías, subcategorías así como los incidentes presentados; así mismo, se planteó culminar con el desarrollo de las mismas premisas anteriores.

\section{REFLEXIONES FINALES}

El empleo de las TIC como estrategia de Apropiación de la Lectura en Niños de Primaria, requiere tanto de la gestión de recursos tecnológicos como de la formación docente para así poder darle el uso efectivo a los mismos.

Por ello entonces es importante el aprovechamiento de los recursos digitales y la infraestructura tecnológica para poder darle un claro conocimiento a su funcionamiento y poder visualizar todas las bondades y fines pedagógicos que los mismos representan. De la misma manera surge la necesidad de realizar un diagnóstico en los estudiantes al inicio de año escolar para poder detectar las competencias que posean cada uno en cuanto al uso de las herramientas tecnológicas y poder así determinar las estrategias de mediación lectora que se van a utilizar en las aulas de clases.

También es pertinente señalar que para la implementación de un plan escolar TIC hay que crear las condiciones necesarias para que el mismo pueda ser desarrollado exitosamente, de lo contrario se correría el riesgo de realizar una inversión estéril en una infraestructura tecnológica que se limita a ocupar un espacio escolar que no es aprovechado para la mediación tecnológica de los aprendizajes.

Finalmente, los docentes cada vez tenemos que ser más conscientes, como nos lo recuerda (Gros, 2000, p.11), "que tan solo el uso intensivo de las tecnologías es lo que realmente conlleva su apropiación. Que realmente estarán integradas al quehacer educativo, al quehacer áulico, cuando dejemos de hablar de ellas, cuando sean algo invisible". Para ello hay que tener en cuenta las desigualdades socioeconómicas culturales que existen en el mundo, y es aquí donde los docentes deben jugar para así poder sacar el máximo provecho de las nuevas tecnologías, pero teniendo plena conciencia de lo real, lo posible y lo necesario 


\section{REFERENCIAS CONSULTADAS}

1. Delors J. (1997) La educación encierra un tesoro [documento en línea]. Disponible: http://www.unesco.org/education/pdf/DELORS_S.PDF [Consulta: 2019, julio 3]

2. Gros Salvat, B (2010). El ordenador invisible. Hacia la apropiación del ordenador en la enseñanza. Barcelona: Editorial Gedisa.

3. Hepp P., (2011). El desafío de las TIC como instrumento de Aprendizaje. Ponencia del Seminario Internacional Cómo las TIC transforman la escuela. Unicef. Buenos Aires. Argentina.

4. López Espinoza, D(2019) Tendencias Pedagógicas y Herramientas Digitales en el Aula: Una Propuesta para su Inclusión Adecuada, en la Carrera de Contabilidad y Auditoría de la Universidad Católica De Cuenca, Extensión La Troncal. Articulo. KOINONIA. Revista Arbitrada Interdisciplinaria de Ciencias de la Educación, Turismo, Ciencias Sociales y Económica, Ciencias del Agro y Mar y Ciencias Exactas y aplicadas. Año IV. Vol IV. Nㅜ․ Enero - junio 2019.

5. Lugo T., y Kelly V., (2011). La Gestión de las TIC en las escuelas: el desafío de gestionar la innovación. Ponencia del Seminario Internacional, Cómo las TIC transforman la escuela. Unicef. Buenos Aires. Argentina.

6. Mercado J. (2010) El diseño metodológico, [documento en línea] disponible en: http://docentes.uto.edu.bo/jmercador/wpcontent/uploads/TEMA_9_EL_DISE\% C\%91O_METODOLOGICO_[Modo_de_compa.pdf, [Consulta: 2019, agosto 30].

7. Ministerio de Educación Nacional de Colombia (MEN 2013). Competencias TIC para el desarrollo profesional docente. Revista en línea Colombia Aprende. W.W.W mineducacion.gov.co.

8. Molano G., Quiroga Á., Romero A., Pinilla C. (2015) Mediación tecnológica como herramienta de aprendizaje de la lectura y escritura, Alteridad. Revista de Educación, [revista en línea], disponible en: http://dspace.ups.edu.ec/handle/123456789/13761

9. Pérez, Cortés, S (2006). La travesía de la escritura. De la cultura oral a la cultura escrita. México: Taurus.

10. Rodríguez J. (2013) paradigmas, enfoques y métodos, [documento en línea], disponible en: https://ce16ba11-a-62cb3a1a-ssites.googlegroups.com/site/jesuscamposg19/assignments/investigarparatran sformaronfalara/PARADIGMAY\%20METODOS\%20DE\%20INVESTIGACION 
\%20EDUCATIVA\%20\%281\%29.pdf?attachauth=ANoY7cqKmcFkQc=0 [Consulta: 2019, agosto 30]

11.Sáez Vacas, F (2004). La Red Universal Digital, [en línea]. Cap. 5 de "Más allá de Internet: La Red Universal Digital. X-Economía y Nuevo Entorno Tecno Social". Extractos en formato pdf de algunos capítulos de la edición personal del propio autor. http://www.gsi.dit.upm.es/ fsaez/int//Red UniversalDigital/libroExtractospdf/reduniversasldigitalpaginaweb.pdf.

12. Tobón S., (2011). Formación Integral y Competencias. Ecoediciones, 3ra. Edición. Bogotá, Colombia.

13. UNESCO, (2013). Enfoques estratégicos sobre las TICS en educación. Serie Bibliotecología y gestión de información. Santiago de Chile.

14. Yanes, J. (2011). Las TIC y la crisis de la educación. Biblioteca digital virtual.

\section{REFERENCES CONSULTED}

1. Delors J. (1997) education contains a treasure [online document]. Available: http://www.unesco.org/education/pdf/DELORS_S.PDF [Consultation: 2019, July 3]

2. Gros Salvat, B (2010). The invisible computer Towards the appropriation of the computer in teaching. Barcelona: Gedisa Editorial.

3. Hepp P., (2011). The challenge of ICT as an instrument of Learning. Presentation of the International Seminar How ICT transforms the school. UNICEF Buenos Aires. Argentina.

4. López Espinoza, D (2019) Pedagogical Trends and Digital Tools in the Classroom: A Proposal for Adequate Inclusion, in the Accounting and Audit Career of the Catholic University of Cuenca, La Troncal Extension. Article. Koinonia Interdisciplinary Arbitrated Journal of Education, Tourism, Social and Economic Sciences, Agro and Sea Sciences and Exact and Applied Sciences. Year IV Vol IV. No. 7 January - June 2019.

5. Lugo T., and Kelly V., (2011). ICT Management in schools: the challenge of managing innovation. Lecture of the International Seminar, How ICT transforms the school. UNICEF Buenos Aires. Argentina.

6. Mercado J. (2010) The methodological design, [online document] available at: http://docentes.uto.edu.bo/jmercador/wpcontent/uploads/TEMA_9_EL_DISE\% C\%910_METODOLOGICO_[Modo_de_compa.pdf, [Query : 2019, August 30]. 
7. Ministry of National Education of Colombia (MEN 2013). ICT skills for professional professional development. Colombia Aprende online magazine. W.W.W mineducacion.gov.co.

8. Molano G., Quiroga Á., Romero A., Pinilla C. (2015) Technological mediation as a learning tool for reading and writing, Alterity. Education Magazine, [online magazine], available at: http://dspace.ups.edu.ec/handle/123456789/13761

9. Pérez, Cortés, S (2006). The crossing of writing. From oral culture to written culture. Mexico: Taurus.

10. Rodríguez J. (2013) paradigms, approaches and methods, [online document], available at: https://ce16ba11-a-62cb3a1a-ssites.googlegroups.com/site/jesuscamposg19/assignments/investigarparatran sformar-pnfalara /PARADIGMAY\%20METODOS\%20DE\%20INVESTIGACION\%20EDUCATIV A\%20\%281\%29.pdf?attachauth=ANoY7cqKmcFkQc=0 [Consultation: 2019, August 30]

11.Sáez Vacas, F (2004). The Universal Digital Network, [online]. chap. 5 of "Beyond the Internet: The Universal Digital Network. X-Economy and New Techno Social Environment ". Excerpts in pdf format of some chapters of the author's personal edition. http://www.gsi.dit.upm.es/ fsaez/int//Red UniversalDigital / bookExtractospdf / reduniversasldigitalpaginaweb.pdf.

12.Tobón S., (2011). Integral Training and Competencies. Ecoediciones, 3rd. Edition. Bogota Colombia.

13. UNESCO, (2013). Strategic approaches to ICT in education. Library and information management series. Santiago of Chile.

14. Yanes, J. (2011). ICT and the education crisis. Virtual digital library. 